\title{
Implementing public health at the national level
}

Major public health problems require major solutions. To be successful, solutions need to be delivered at a number of levels. The English white paper Saving lives: our healthier nation" stated "We want to see healthier people in a healthier country. People improving their own health supported by communities working through local organisations against a backdrop of action by the Government".

To illustrate this backdrop of actions, we can look at nutrition, where the concern might be an insufficient or an inappropriate diet. To assess the need for action a number of questions must be asked. Do people understand the basis of a healthy diet, do they have access to it, can they afford it, are there the right incentives for the food industry and agriculture to produce the right food at the right price, are these linked to sustainable development? The responses to these questions indicate the many levels of potential action. For example, education and access might be tackled at the local level, but reducing poverty and providing incentives for agriculture require national action. While public health programmes must engage communities and individuals, to be successful they need effective underpinning policies. These should not be solely health policies, but must tackle cross government issues that affect both the individual and the wider environment.

Key elements of the national response must be an analysis of the problem, determination of the overall approach, engagement of other players across government and identification of policies that will support change at the local level. To be effective, such policies should be developed in an interactive way with the field, to avoid the "top down" approach, which can result in policies that are impractical to implement at the local level.

Cross government policies can be complex, often with competing priorities and the health gain is not always clear. Transport policies, which encourage walking and cycling and discourage car use perhaps through taxation, may also create lack of access to services, employment or social activities in rural areas. The development of health impact assessments (HIA) should help work through these complexities, but the methodology is at an early stage. As anticipated outcomes are often long term, HIAs depend on many assumptions for which the evidence is limited.

Policies should be backed by a range of supporting features. First among these is leadership. For those working on public health issues in the National Health Service, their work is much easier if those running the service place a high priority on public health measures. Empowerment is stronger if the national lead is clear.

Governments can set national targets. Targets may have a distorting effect, as they may channel action in a limited way, but their public nature can help to sustain action. For example, in England there are high levels of teenage conceptions. The government as a whole, not just the department of health, has committed itself to a goal of halving the rate of conceptions among under $18 \mathrm{~s}$ in England by 2010, which requires a continuing input from a number of government departments.

Work must be supported by resource allocation. This can be in the general distribution of funds, such as increased funding for areas that have the greatest need, both in health services and in other public programmes. It may also be specific funding for individual programmes, such as smoking cessation or reducing fuel poverty.

The centre can place specific requirements on those running local services to deliver programmes and monitor their performance. The behaviour of those running such local services is affected by how they are judged. The requirements set may support effective joint working at the local level, but may encourage behaviours that detract from such partnerships. For joined up local working, we need to achieve joined up national working. Similarly, local targets and requirements will affect where local managers place their priorities. For example, if the main requirements are about health service delivery, rather than programmes of prevention, service delivery will be the main effort. This affects those working in preventive services. The monitoring of performance should not mean second guessing those working in the field. The national role should be in requiring what needs to be achieved, not how it should be done. Delivery is better determined by those experienced in running programmes or services.

Those working at a national level can support those running programmes by coordinating efforts. For example, in England, a Forum of Non-Government Organisations has been set up. The Forum aims to enable NGOs to advise the government on implementing its public health policies, and to facilitate the coordination of their work to achieve maximum impact. The centre can fund pilot programmes, and disseminate good practice.

Essential underpinning programmes for public health require national action. Monitoring public health and the delivery of public health programmes needs information, and data collected in a standard and timely way. The development of information systems should be led nationally, although, as in the development of policies, an interactive approach is essential if the information is to be of use to those running programmes. Data may be analysed at all levels, but national analysis, to enable comparisons, to link datasets, and to ensure continuity is also vital. As well as routine data collection, national surveys can inform programmes. For example, in England there is an annual National Health Survey, which includes core sections each year, but has additional sections to consider particular problems.

Public health should be based on sound evidence. Funding can be allocated for research, both through national funding streams and through the influencing of the main research funders such as the major charities. Public health programmes are labour intensive, requiring input from many professional groups, and there is a national role in the development of a workforce strategy, backed by funding and training programmes.

Programmes may also need to be backed up by legislation. For example, legislation on industrial and vehicle emissions will contribute to health protection, legislation on tobacco advertising will contribute to health promotion. Increasingly, the legislation is international, and for the UK, working within the European Union is important. Even where legislation is not a factor, working with partners in organisations such as the World Health Organisation can affect international approaches to tackling problems that affect each country domestically. An obvious area is in the field of communicable diseases, where increased globalisation has affected the spread of diseases, and associated problems such as antimicrobial resistance. 
In summary, for public health programmes to be successful, there has to be an active contribution at the national level. First and foremost that should be to develop policies, not just in public health departments, but across government, and to provide leadership. These need to be backed up by resource allocation, targets and performance management, by information, research and workforce strategies, by supporting and coordinating those in the field, and where necessary by legislation. Increasingly, this needs to be linked to international action.

PAT TROOP

Deputy Chief Medical Officer,

Department of Health

1 Department of Health. Saving lives: our healthier nation. London: The Stationery Office, 1999

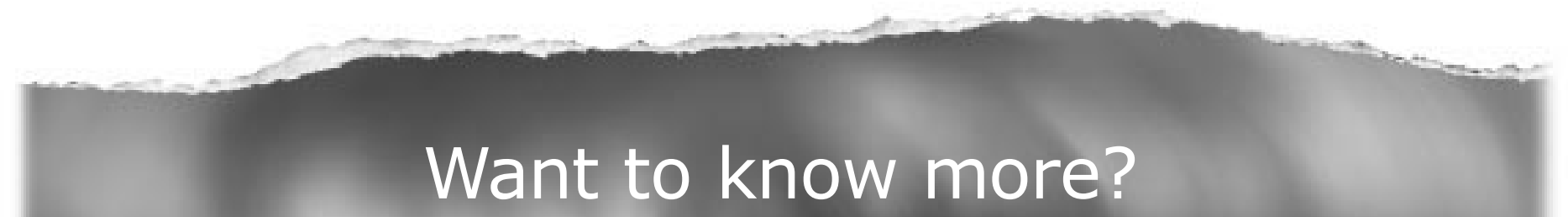

Data supplements

Limited space in printed journals means that interesting data and other material are often edited out of articles; however, limitless cyberspace means that we can include this information online. Look out for additional tables, references, illustrations.

www.jech.com 